\title{
Self-awareness of Dual Roles When Using the CIT (Critical Incident Technique): Opening Pandora’s Box?
}

\author{
Lianne Britten \\ Macquarie University, NSW, \\ Australia
}

\author{
William Borgen \\ University of British Columbia, BC, \\ Canada
}

\author{
Mark Wiggins \\ Macquarie University, NSW, \\ Australia
}

\begin{abstract}
The issue of conducting ethical research that considers the wider impact on the participant is not a new idea in academia. It is the constant "elephant in the room” that exists for any researcher who works with people and this is accentuated when working with vulnerable populations. This article is written as a cautionary note and offers an ethical and moral means of conducting research in the moral quagmire of honoring people's stories, words, behaviors and thoughts. The purpose is for the researcher to note the possible impact that the telling of such stories may have not just on the storyteller, but also on the listener. It is meant to serve as one of the ways to minimize harm to participants and researchers, and maximize the potential of the widely used qualitative research tool and the CIT (critical incident technique). Following in the footsteps of the pilot study by Butterfield, Borgen, Maglio, and Amundsen (2009) regarding the impact of a qualitative research interview on participants, combined with previous post-modern analyses, it is clear that any type of research can have an impact on participants. This article positions itself as a means of advocating that a researcher working with potentially vulnerable populations should wear "dual hats" when conducting research and, at all times, should be aware of the impact upon participants during the research process. Further, it explores the need to the potential impact first and foremost in the mind of the researcher prior to embarking upon any research with vulnerable and special populations.
\end{abstract}

Keywords: CIT (critical incident technique), immigrant, career, youth, special populations

\section{Origins of CIT (Critical Incident Technique)}

The CIT is a qualitative research tool that was introduced after World War II, growing out of the aviation psychology programme for the US air force (Flanagan, 1954). Its continued popularity, deployment and development have been driven by a desire, through organizational and industrial psychology, to identify key personnel for jobs, methods of determining job requirements and effective system design.

Flanagan (1954) initially established the CIT as a set of procedures to enable the systematic analysis of effective and ineffective performance of pilots. The five steps include: (1) to identify the aim of the study and the research question; (2) to identify the types of incidents to be collected; (3) to identify the means of data collection; (4) the analysis of the data; and (5) the categorization and codification of the data. More generally, the CIT represents a set of procedures for systematically identifying behaviors that contribute to the success or failure of individuals or

Lianne Britten, Ph.D. candidate, Department of Psychology, Macquarie University.

William Borgen, Ph.D., professor, Department of Educational and Counseling Psychology, University of British Columbia. Mark Wiggins, Ph.D., associate professor, Department of Psychology, Faculty of Human Sciences, Macquarie University. 
organizations in specific situations (Butterfield, Borgen, Maglio, \& Amundsen 2009). Since its inception, this technique has traditionally been applied with little regard to the psychological impact of using this technique upon the individuals who are being asked to recall events (Butterfield et al., 2009; Bradbury-Jones \& Tranter, 2008).

CIT has, typically, been used as a means of collecting data from an exclusively cognitive perspective. The main premise that underlies CIT is the recall and verbalization of a critical incident (Butterfield et al., 2009). Such incidents are typically thought to constitute memorable experiences. However, the traditional method of employing CIT as a benign method of extracting information from "experts" in the field and not paying heed to the possible psychological impact on the participant belies the fact that the critical incidents to which respondents refer, may trigger significant psychological distress. It is worth noting that Butterfield et al. (2009) underscored this potential and suggested that ethical mandates need to be kept at the forefront of any research to minimize damage or "harm" to participants. Ethical mandates pertaining to research are clear in their recommendation to researchers to place respect, reciprocity, equality, survival and protections, responsibility, spirit and integrity at the centre of any research project. However, the traditional approach to CIT is one that involves the collection of direct observations of human behavior "in such a way as to facilitate their potential usefulness in solving practical problems” (Flanagan, 1954, p. 327).

Flanagan (1954) defined the CIT as a qualitative research method designed to enable the recall and description of critical incidents, the data from which could be used to determine optimal strategies to fulfill job requirements, such as personnel selection, job roles/responsibilities and identifying errors. Originally, Flanagan defined an incident as "any observable human activity that is sufficiently complete in itself to permit inferences and predictions to be made about the person performing the act” (p. 327). Norman, Redfern, Tomalin, and Oliver (1992) extended this definition in the context of CIT by suggesting that the term "critical incident" refers to a defined event, by a person involved, who is enabled to make an evaluation of the positive or negative impact that the incident has had upon them or their situation. Further, Flanagan (1954) concluded that exhaustiveness or redundancy is needed to have been achieved for the event to be classified as a true "critical incident". Redundancy is achieved when there are no new critical incidents mentioned by the participants, or when one participant exhausts the category by no longer mentioning that category throughout their interview (Butterfield et al., 2009).

There are a number of steps associated with the process of CIT, the first of which is to identify the aim of the study and the research question. To help ensure clarity with regard to the research question, it is important to be clear about the aim of the research. According to Butterfield, Borgen, and Amundsen (2005, p. 478), "Understanding the general aim of the activity is intended to answer two questions: (1) What is the objective of the activity; and (2) What is the person expected to accomplish who engages in the activity?”. This initial step is critical in ensuring that the original aims are adhered to. If there is little clarity regarding the research question and the aims of the research, then the project itself will not be able to capitalize on the nature of "critical incidents". If there are no clear sets of events or behaviors upon which the project is based using CIT, then the focus will be lost and the project can lose direction.

The second step in CIT is to identify the types of incidents to be collected. The use of CIT is optimal when specific incidents, thoughts and behaviors are collected from those specifically able to judge if and how these incidents may be significant (Butterfield et al., 2005). If there is no ability to collect "specific" data, then the ability to judge the significance of the incidents is diminished and can lead to a profusion of ideas, categories while the analysis of the data could mean findings that are meaningless or have wide variations.

The third step of the CIT is the means of data collection, and this process can include direct observations, record forms, open-ended questionnaires, in person open-ended interviews, individual, or group interviews 
(Flanagan, 1954). While there are some disadvantages associated with open-ended interviews, including the possible distortion of events from a retrospective perspective of a situation, it is possibly the most frequently used method of CIT data collection (Angelides, 2001; McCormick, 1996; Britten \& Borgen, 2010; Butterfield, et al., 2005b; Butterfield et al., 2009).

The flexibility of CIT is an advantage in collecting naturalistic data, since the optimal sample size can be determined by the number of critical incidents, rather than by the actual number of participants in the study. However, this can also lead to confusion, as some projects may have as few as eight participants and yet record over 550 incidents (Britten \& Borgen, 2010) or may include multiple participants with relatively few incidents (Butterfield et al., 2005b; Butterfield et al., 2009). According to Flanagan (1954), Woolsey (1986) and Butterfield et al. (2005a) and Butterfield et al. (2009), there is no set guideline as to how many incidents are sufficient for the purposes of determining a critical incident. Rather, it is more important that there is sufficient detail relating to the incident in question.

The fourth step in the CIT is the analysis of the data. As outlined by Flanagan (1954), this step is seen as the most difficult and the most important in the CIT process. Woolsey (1986) and Butterfield et al. (2005) and Butterfield et al. (2009) concurred noting that the analysis of the data is both time consuming and comprehensive. To maintain validity, large amounts of data must be analyzed, cross-checked and coded rigorously and with expert attention to categorization and codifying.

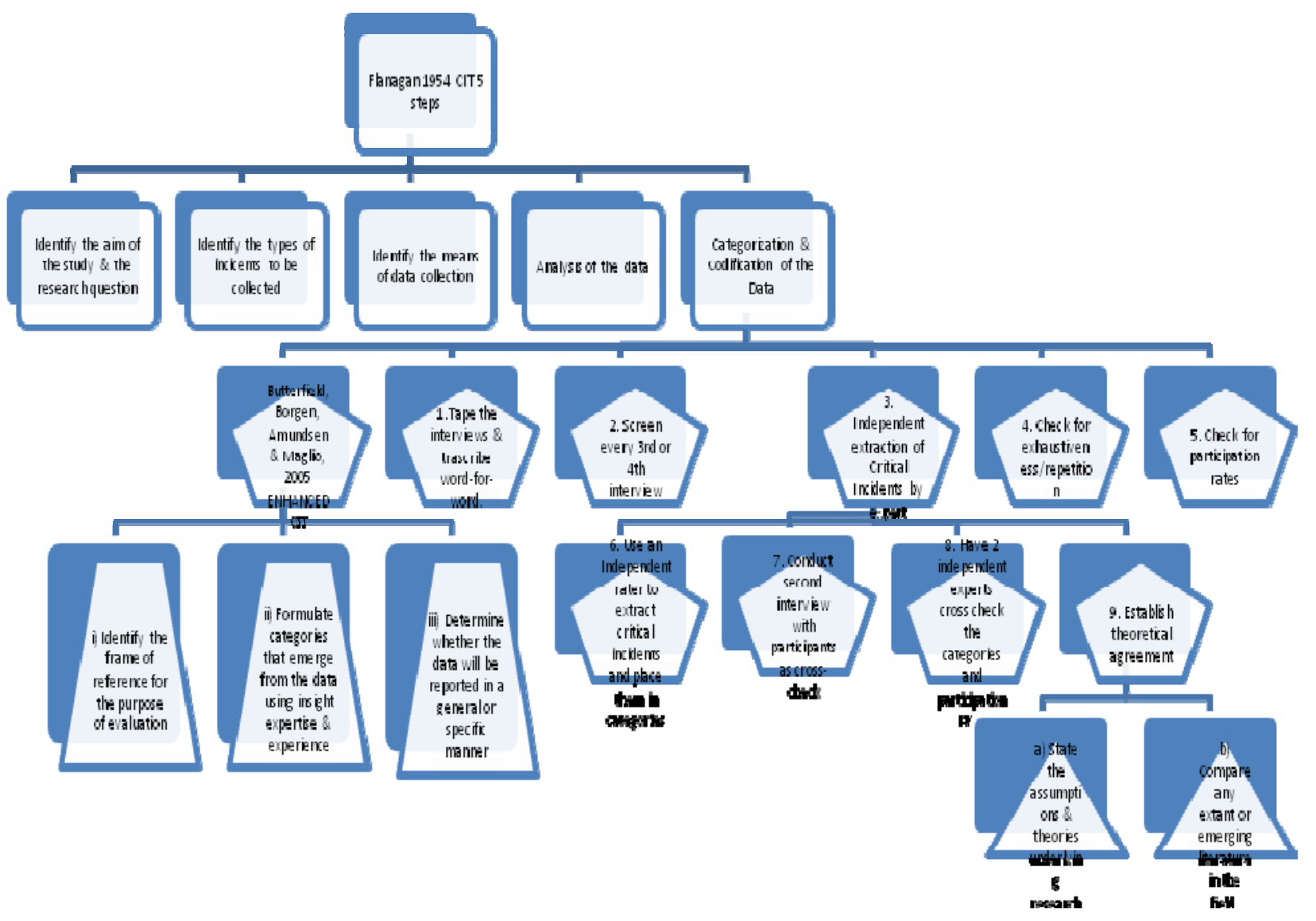

Figure 1. Summary of CIT and enhanced CIT. 
Creating a categorization scheme and codifying multiple incidents within these categories involves a three-tier process as outlined by Butterfield et al. (2005). Firstly, it involves identifying the frame of reference for the purpose of evaluation. Secondly, it is necessary to formulate categories that emerge from the data and which require insight, expertise and experience on the part of the analyst. Such insight, expertise and experience are difficult to acquire and it is for these reasons that vast amounts of data are often accumulated.

The final step in categorization and codification is to determine whether the data will be reported in a specific or general manner. These decisions and the ultimate reporting of the data (the fifth step) can lead to wide variations in results and may also cause confusion for the researchers following the CIT. There are distinct differences in choosing to report the data in a more general manner, rather than focusing on specific categories and limiting the data to that area (Butterfield et al., 2009). Yet, it is this openness and flexibility which gives CIT distinct advantages over some other techniques with regard collecting, otherwise unknown or unstudied phenomena, while honoring the participant's voice and acknowledging the vast diversity of cultural and ethnic people.

The utility of CIT lies in its flexibility and the scope of the tool to inquire about a plethora of phenomena, behaviors and their impact. According to Flanagan (1954, p. 335), it "does not consist of a single rigid set of rules governing such data collection. Rather, it should be thought of as a flexible set of principles that must be modified and adapted to meet the specific situation at hand”. Flanagan (1954) defined a number of steps in the CIT methodology for the purposes of replication and consistency in the process of conducting research with the CIT method, and these are the basis for suggestions by Butterfield et al. (2005a), for the application of ECIT (enhanced critical incident technique).

Due to a number of criticisms and limitations with regard to the validity of the CIT, Butterfield et al. (2009) added nine steps for the purposes of assessing the credibility of the outcomes of CIT. These new additions have become known as the ECIT which is a development of CIT introduced by Butterfield et al. (2005b) for the purposes of adding some standardization to the tool and enabling credibility checks, and is one solution to some of the issues associated with using the CIT.

One of the criticisms of CIT has been the potential lack of validity of the tool in terms of the meaning of what constitutes a "critical incident" (Bradbury-Jones \& Tranter, 2008). This criticism stems from the flexibility of the term, but it is also a question based on the reality of the difference between the perceived impact of the critical incident on both the phenomenon being studied and the outcome of the critical incident. The question becomes more about how we know, if an event actually fulfils the definition of the term "critical incident". Both Flanagan and Butterfield recommend a more positivist means of analyzing and collecting data, which attempts to balance this uncertainty with a number of credibility checks as advocated by Butterfield et al. (2005) in the ECIT. The credibility checks are added to the "analysis and the reporting of the data" stage of CIT.

The first of the credibility checks in enhanced CIT is to tape the interviews, and then to have these interviews transcribed verbatim. These interviews are then screened by an expert on a regular basis. Butterfield et al. (2005) suggested that every third or fourth interview be included as a means of ensuring that the CIT protocols are followed and that the interviewer is not "leading" the participant. This check not only functions as a means of ensuring that correct protocols are being followed, but it also serves as a measure of screening out "white noise" or, incidents not critical to the phenomena, and focusing on the central elements of the research question. This does not eliminate the possibility of further explication and exploration of the phenomena, but with the word-for-word transcription and screening by an expert in the field, it strengthens the ability of the 
researcher to hone in on the more relevant concepts and events so that incidents can fit the definition of a "critical incident” more clearly.

Butterfield et al. (2005) also suggested that there should be an independent extraction of the critical incidents from the data collected by an expert in the specific field of research, to determine whether the incident fits a critical incident or rather a "wish list" item. The fourth credibility check is listed as checking for exhaustiveness, which is then followed by the fifth check for participation rates.

The sixth credibility check involves the use of an independent rater, who also extracts critical incidents and places them independently into the categories. This occurs by having the independent rater, randomly choosing $25 \%$ of the incidents and placing them into categories. This should be matched against the researcher's match rate for a rate of not less than $80 \%$ (Andersson, \& Nilsson, 1964).

The seventh credibility check is the second interview which is the cross-check of the incidents and the categorization of the incidents by the participant him/herself. The purpose of this check is to ensure that the participant's words have been honored and allow participants the opportunity to correct, edit or delete those incidents that they consider that feel did not represent their story correctly.

The eighth credibility check involves having two independent experts in the field cross-check the categories and participation rates. Finally, the ninth step that Butterfield et al.’s (2009) suggested is a need for theoretical agreement in two ways. The first is to state the assumptions and theories underlying the research and the second is to compare any existent or emerging literature in the area where the study is being conducted.

The additional steps that Butterfield et al. (2009) have added to the CIT not only add some form of standardization to data collection, but they also form a clear guideline for researchers. This helps to clarify the research protocol and maintain a consistent approach to the CIT interviews. However, while noting these changes, it is important to acknowledge that, even with these additions to the CIT, there remains a perception of a benign approach to data acquisition. It is also necessary, as noted previously, to understand that this assumption is not possible when using narrative as a form of research inquiry (Butterfield et al., 2009).

\section{Advantages of the CIT}

CIT can be used for a variety of study purposes and across a wide variety of areas (Butterfield et al., 2005b, Butterfield et al., 2009; Schluter, Seaton, \& Chaboyer, 2008; Britten \& Borgen, 2010; Bradbury-Jones \& Tranter, 2008). The foci range from looking at helping and hindering factors in the workplace, to studying effective and ineffective ways of behaving, collecting functional or behavioral descriptions of events or problems, or determining factors that are vital to an event or activity (Butterfield et al., 2009; Bedi, Davis, \& Williams, 2005). In all of these studies, however, there is a need for human participants to include their insight and expertise into the area of interest.

This opportunity for self-insight is one of the main advantages of the CIT, but it is also an area for caution. It is often necessary when using this technique to go beyond looking at the "critical incident" as separate from the person talking about it and rather, to look at the wide ranging impact of such interviews and discussions on the individuals participating in such studies. This is a result of the unpredictable nature of storytelling. In narratives, the storyteller may divulge unexpected or possibly traumatic information, even in seemingly "safe" research areas. It is often not possible to predict the ways in which oral narratives can impact the participant. Whenever conducting research with vulnerable populations, the use of the CIT brings with it a need for constant awareness of any changes in demeanor or affect on the part of the participant. This is necessary to 
ensure that any possible harm to the participant is minimized and appropriate referrals or actions are taken to ensure the participant's safety (Butterfield et al., 2009).

Another advantage of the CIT is its ability to be used with cross-cultural populations. There is noteworthy evidence of the need for research within non-mainstream communities to be not only empathic, ethical and respectful, but also for it to follow epistemology that is regarded as central to the cultures that are being studied (Kingsley, Phillips, Townsend, \& Henderson-Wilson, 2010; McKeough et al., 2008; Hallet et al., 2008; Britten \& Borgen, 2010; McCormick, 1996). For example, McKeough et al. (2008) argued that storytelling fits with aboriginal epistemology and is not only a traditional educational tool but is also culturally relevant. Similarly, Kingsley et al. (2008) recommend using research methods which consider notions of negotiation, mutual understanding, respect, recognition, involvement, shared ownership, reciprocity, skills development and methodological flexibility.

Using the CIT in a narrative format with semi-structured interview questions, and working in conjunction with an organization to oversee and help guide the process helps to fit with non-mainstream or cross-cultural epistemologies that value storytelling and oral narratives. Within indigenous cultures, storytelling is a traditional teaching tool, and across many ethnic and migrant cultures, oral history is also familiar and culturally relevant (McKeough et al., 2008; McCormick, 1996; Angelides, 2001; Pedersen \& Barlow, 2008). Further, through collaboration with organizations and cultural experts, the researcher is made more aware of the various cultural protocols and ethnic traditions that may impact the participant. This awareness is important for conducting culturally appropriate and sensitive research (Day \& White, 2008).

\section{Flexibility}

The flexibility of the multiple methods of data collection and analysis in CIT adds options for data collection that can overcome some of the limitations associated with standardized tools. Standardized tools have been seen by non-mainstream populations as failing to account for non-standardized populations and are not seen by many cultures as being valid in terms of their own cultural and traditional mores (Kingsley et al., 2010; Pederson \& Barlow, 2008). For example, the use of open-ended semi-structured interview questions allows for considerable scope in the researcher's ability to probe further into the incident, thereby ensuring that additional and meaningful data are acquired.

In a cross-cultural research design, it is important to note that individual, social and cultural variations are a part of the design (Kim \& Berry, 1993). Using an experimental research method design works well for some basic psychological processes but, according to Wundt (1916), it is not appropriate for use with processes that are shaped by language and culture. In cross-cultural and indigenous research, it is necessary to incorporate culture, speech, myth, art, religion, literature, laws, customs and morality to understand the culture's "human qualities” (Kim \& Berry, 1993; Danziger, 1979). Further, not all psychological processes are universal, and not all cultures have an individualistic cultural base. Therefore, using standardized tools fails to capture the variations of non-standard populations (Wundt, 1916; Danziger, 1979; Kim \& Berry, 1993).

When studying cultures, it is also necessary to understand a particular culture from its own frame of reference. This frame of reference includes historical and comparative analyses of symbols, culture, language and other factors. Without some referencing from within each culture being examined, it will not be possible to understand the underlying dynamics of the human psychological processes within that culture (Berlin, 1976; Kim \& Berry, 1993). The failure to account for these cultural variations can lead to cultural "arrogance" and 
distortions of the research findings through researchers using judgments based on their own standards of reference, rather than an understanding of the cultures which they are studying (Wundt, 1916; Berlin, 1976; Kim \& Berry, 1993; Filbert \& Flynn, 2010).

Indeed, the CIT represents an effective tool for generating detailed data and descriptions of phenomena from participants (Angelides, 2001; Butterfield et al., 2005b; Andersson \& Nilsson, 1964). The CIT also has the potential to go beyond traditional means of acquiring data and provide scope for the researcher to probe unknown phenomena in a deep, meaningful and rich context (Butterfield et al., 2009). Finally, CIT is useful for the purposes of achieving exhaustiveness and giving a more complete picture of the phenomenon being studied (Butterfield et al., 2005b; Andersson \& Nilsson, 1964).

Rich and contextualized data is a necessary component of research when working with particular populations, and the CIT provides great scope for the researcher to achieve the goals of ethical research while honoring voice and tradition. For cross-cultural research to achieve acceptance in the communities affected and included in the work, the researcher must also be accepted. Given the history of imposition, prejudice and oppression for many of these non-mainstream cultures with regard to research practices and governmental policies, it is important that all participants from these vulnerable populations be given respect, and that the researcher be accountable for honoring words, tradition and cultural mores (Kingsley et al., 2010; Filbert \& Flynn, 2010). It is, therefore, important to recognize the voice of the participants and operate such research in an ethical manner so that when working with certain populations and research questions, the lived experience of the participant is explored.

\section{Risks}

CIT research has been used for almost six decades and has been based on the perhaps implicit belief that the role of the research process is rather "benign". The researcher is typically seen as someone who enters an organization or environment, extracts information from personnel and then walks away to analyze the information for the purposes of dealing with an issue to improve existing processes. As a result, there is often little consideration given to the reality that the participant imparting information may have been significantly impacted by the nature of research process. For example, even in the case of a topic like human error, significant psychological distress may be elicited in the recall and recounting a critical incidents in which human error featured.

The risks of exploring such detailed memories mean that traumatic triggers may be exposed (Butterfield et al., 2009). If this is the case, it is necessary for the researcher to be aware and work with the participant to minimize harm and recommend/refer the participant to the necessary point of assistance if required. While both the original CIT and the ECIT have merit, there remains the potential that, while conducting research, one might be driven by theoretical desires without taking into account the underlying experiences of the participant.

Another risk associated with CIT is the reliance of the research technique on memory and recollection. Memory recall is not a "reliable source" of information due to distortion and "rewriting" the history (Flanagan, 1954; Woolsey, 1986; Butterfield et al., 2005a). For this reason, most researchers using CIT try to use events that have occurred in the recent past, and even put a time limit of up to six months on the collection of information (Butterfield et al., 2009). According to Flanagan (1954), the more detailed the recollection of an event, the more accurate the recollection. Therefore, it is important that all the descriptions and explanations surrounding an incident be explored to reduce the impact of memory recall errors (Butterfield et al., 2009), not 
to improve the recall, but to ensure that the details surrounding an event are as detailed as possible to ensure as much accuracy as possible in the retelling of an event.

\section{Bringing Awareness to Ethical Issues}

Ethical codes and personal values of the researcher are key elements of conducting research with vulnerable populations. There are many pitfalls that a researcher can fall into without even being aware, if measures are not taken to ensure that the research is accountable and responsible. Many steps can be taken to limit issues when using a tool such as CIT. The interview itself is one place where ethics can be breached and a researcher must be cognizant of these at all phases of the research project.

According to Flanagan (1954), the skills of the researcher are important to "tease out" specific, accurate and precise descriptions of an incident or behavior. It is important, therefore, for research interviewers to be trained consistently and to have access to specific, expert, and timely feedback with regard the content and conduct of the interviews. This feedback is integral in ensuring that the interviews follow required protocols, question format, guidelines and probing questions. It is also necessary to ensure that the information provided by the participants is as specific as possible in their descriptions of the phenomena, incidents and the behaviors that they felt were either helpful or not helpful to them with regard to the research question and aims. Therefore, the screening of interviews by an expert is an important element of the ECIT's credibility checks for good reason. This screening is based on checking for questioning focus and the absence of leading by the interviewer, as well as reviewing the suitability of the questions and probes by the interviewer. It is necessary in a research project to keep the interviewer, and hence, the interview, on track and focused on the research question.

Interview screening by experts is also a means to ensure that, should any cause for concern for the well-being of the individual participant arise, not just the researcher, but two qualified, expert and registered psychologists, can also add their lens to the issue and make suggestions. Thus, the screening of the interviews and the cross-checking of incident transcription data is not simply a validation technique, but also a means of ensuring that the participants were under the scrutiny of a number of experienced researchers.

In addition to screening, another validity check associated with ECIT concerns the application of the second interview. Flanagan (1954) maintained that the process of data collection should be focused on the participant's perspectives and that the second interview is a means of ensuring this occurs within the research design. Butterfield et al. (2009) discussed the importance of the second interview as: (1) the need for the participant to review the researcher's interpretations of the data; (2) the need for the participant to review the themed categories that were decided upon by the researcher; (3) the need for confirmation that the item was a critical incident or a wish list item; and (4) the need to ensure that the participants' voices have been heard accurately reported and honored.

The second interview is also an opportune time to assess the participants' well-being following the initial recount. The time frame for this second interview can be anywhere from three to 12 months after the first interview, dependent upon a number of factors including the size of the research participant base, the turnaround times for transcription and the analysis of the data itself, as well as the need to complete validation checks for the categories and the placing of incidents within categories. It also allows both the researcher and the participant the scope to monitor the progress and state of the participant should any unforeseen incident trigger a response that the participant may have been unaware of, or unprepared for. Thus, the second interview is an especially important element in the conduct of ethical research with vulnerable populations. 
In addition to these credibility checks, it is important to pay attention to the continued and consistent training of the researcher, following the guidelines established by the ECIT. Ensuring that the researcher and interviewers have experience with the CIT contributes to ensuring a consistent approach to not only the collection and analysis of data but also to the well-being of the participants. This is not always possible, since the pool of participants and the size of the database may sometimes require additional personnel for completion. However, it should be noted that the requirement for additional personnel can be addressed by paying close attention to the training of research teams, as well as adherence to the recommended ECIT protocols, including screening of interviews and cross-checks, as these measures can mitigate the potential for a lack of consistency with regard to interviewing techniques, data collection and analysis.

One other means of reducing possible harm with regard to the use of the CIT is to ensure that participants are pre-screened as to their appropriateness for inclusion in the study. Pre-screening performs two roles. Firstly, it ensures that the participants who are going to be interviewed actually fit the criteria for the research question, and secondly, it ensures that potential participants are screened for specific vulnerabilities and/or mental health issues that may be triggered during an interview. This then gives the researcher the opportunity to offer counseling for prospective participants deemed needing assistance, and also screen for participants for whom the experience might be seen as unhelpful. Pre-screening is also an opportunity for the researcher to inform prospective participants of the nature of the research and answer any questions that they may have prior to participation, to fulfill ethical requirements of informed consent (NHMRC (National Health and Medical Research Council), 2003; AIATSIS (Australian Institute of Aboriginal and Torres Strait Island Studies), 2000).

In addition to the need for voice, there is a need to maintain participant confidentiality and understand the need for circumspection with regard to the identification of locales, communities and even the ethnic identities of participants in these specific types of research projects with special populations. It is imperative that any identification of third parties, geographic locations or any possible identifying features be removed from the data reported to maintain the anonymity of the participants and their respective communities or people.

This key point regarding the maintenance of confidentiality is significant when dealing with reporting back to communities and organizations. For example, Flanagan (1954, p. 355) stated that "The research worker is responsible for pointing out not only the limitations but also the degree of credibility and the value of the final results obtained". For these reasons, the second interview can also be used as a means of ensuring that the participant accepts the manner in which the data are reported and whether words such as "mother" or "father", etc. might need to be removed and replaced with family member or some other less specific term. These small but important details can add to the value of the research when reporting back to small communities, as it helps to limit the issue of "who" said "what" and instead, lead to the focus towards the identification of solutions.

To fit with the notion of exploring unknown phenomena in depth and understanding the cultures from their own frames of reference, it can be useful in CIT studies to use basic framing questions for the research project. Such guiding questions for the purposes of collecting data for any CIT study can be based on such questions as: Is this capturing the essence of what preceded or contributed to an incident or phenomena?; How did the participant act or not act with regard to the incident; and What was the outcome of those actions/inactions? The final question is based on the point of view of the participants and how this may have helped or hindered them in their journey (Schluter, Seaton, \& Chaboyer, 2008; Butterfield et al., 2005a). This question is aptly addressed by the intention of the CIT to "explore an unknown area of research" (Britten \& Borgen, 2010, p. 106). CIT offers an unparalleled means of gaining insight into rarely studied experiences, 
particularly when working with populations whose voice has rarely been heard or accepted.

In research involving personal experiences, the importance of allowing ample time for the participants to tell their stories must be balanced against the need to be aware of the duress and/or endurance of the participants. Many interviews will last for extended periods of time. Ethically, however, it is necessary to assess the mental and physical state of participants both verbally as to their ability and willingness to continue. Following the assessment, the interview needs to be drawn to a close with the reminder that there is a second, check-in interview whereupon important themes, ideas and events could be revisited. For many of the participants, the process will be considered not only enjoyable, but also a rare opportunity for them to speak about things that they had not been able to do so previously.

It is also important to inform the participant of the recourse to counseling should that be required for any reason. Advice should be provided at the beginning of the first interview, at the signing of the consent form and again at the end of the completion of the first interview. Further, at the check-in second interview, the author should ask the participants, in general, how they feel about their participation and reiterate the availability of counseling.

\section{Conclusions}

In conclusion, the CIT is an extremely useful and flexible tool that provides an unparalleled opportunity for researchers to explore and explain previously unstudied phenomena or add to academic research in a rich and in-depth manner. The ECIT is a means of ensuring that the research conducted is standardized and able to be assessed for credibility through a number of protocols. However, both of these tools have limitations. Implicitly, both Flanagan's original CIT and the ECIT as proposed by Butterfield et al. (2009), are consistent with a more positivist tradition. It is important for the researcher working with sensitive and special populations to mediate between the positivist tradition of imposing a theoretical framework upon the research, with the need for a lens that uses grounded theory or phenomenology to explore the area.

It is the continued paradox of the perception of "benign" research being able to be conducted with an objective analysis of what can often be very personal and "critical" events to the respective individual participants. Therefore, it is necessary to note that, while the CIT and ECIT offers an unparalleled means of gaining previously unknown data, there is also a need to balance adopting a "top-down" data acquisition format with a "bottom-up" analysis of those same data. The compromise is the acceptance of the structure for a useful protocol and a standard means of acquiring data, while simultaneously adopting a grounded theory perspective in the analysis. The ethical and moral obligations to do in particular environments such as "cross-cultural" research make the imposition of a positivist ideology anathema to the whole notion of grounded theory.

Yet, while conducting research with certain populations, it is important to filter the results through a lens where ethics and moral values suggest that such exploration must be conducted with the intent to provide "voice" to the participants, rather than imposing a theoretical model upon the research. It must be noted that, when using the CIT, as with any research tool, the researcher must be cognizant of possible issues for the participants and walk into the world of the research with dual hats: that of a researcher and that of an ethical psychologist or professional. The need for both hats is evident when working with the nature of narrative stories that may involve some reflection upon events across the lifespan, or of critical importance to the narrator. Therefore, by maintaining an awareness of the nature of critical incidents and their unknowable impacts upon the story-teller, the researcher, who uses CIT, may be enabled to walk the fine line of conducting ethical 
research, while simultaneously exploring what could possibly be information concerning a traumatic nature and affect.

On a final note, it is important to understand that cross-cultural and indigenous research projects be conducted with the idea that there are unique cultures and these do need to be understood within their own frames of reference, but there is also the possibility that from such research may arise some universal truths. It cannot be assumed that a finding from one culture is unique only to that culture. Conversely, it cannot be assumed that these findings can, in any way, be generalized. It can only be posited that there is a possibility of the existence of universal truths. In Australia, we do not have a single mainstream to which minorities or indigenous peoples relate to in a "neat" or compartmentalized manner. To try to understand the mosaic of our multicultural and diverse society, it is, therefore, important to use research designs that "allow" for and include these multiple cultural and traditional variations. The CIT offers one means of looking at the Australian cultural mosaic in a respectful, consistent and open manner that values oral narrative. From such research, it is possible to find new collaborative meanings, phenomena and definitions for populations that do not fit with popularized, mainstream ideas.

\section{References}

AIATSIS (Australian Institute of Aboriginal and Torres Strait Island Studies). (2000). Guidelines for ethical research in indigenous studies. Canberra: AIATSIS.

Andersson, B. E., \& Nilsson, S. G. (1964). Studies in the reliability and validity of the critical incident technique. Journal of Applied Technology, 48(6), 398-403.

Angelides, P. (2001). Using critical incidents to understand school cultures. Improving Schools, 4(1), 24-32.

Bedi, R., Davis, M. D., \& Williams, M. (2005). Critical incidents in the formation of the therapeutic alliance from the client's perspective. Psychotherapy: Theory, Research, Practice, Training. Educational Publishing Foundation, 42(3), 311-323.

Berlin, I. (1976). Vico and Herder: Two studies in the history of ideas. New York, Viking.

Bradbury-Jones, C., \& Tranter, S. (2008). Inconsistent use of the critical incident technique in nursing research. Journal of Advanced Nursing, 64(4), 399-407.

Britten, L., \& Borgen, W. A. (2010). Footprints of color along the career journey. Procedia Social and Behavioral Sciences, 5 , 104-115.

Bryman, A. (1984). The debate about quantitative and qualitative research: A question of method or epistemology? British Journal of Sociology, 35(1), 75-92.

Butterfield, L., Borgen, W. A., \& Amundsen, N. (2005a). The impact of a qualitative research interview on workers' views of their situation. Canadian Journal of Counselling, 43(2), 120-130.

Butterfield, L., Borgen, W. A., \& Amundsen, N. (2005b). Fifty years of the critical incident technique: 1954-2004 and beyond. Qualitative Research 2005, 5(4), 475-497.

Butterfield, L., Borgen, W. A., Maglio, A., \& Amundsen, N. (2009). Using the enhanced critical incident technique in counseling psychology research. Canadian Journal of Counseling, 43(4), 265-282.

Danziger, K. (1979). Origins and basic principles of Wundt’s völkerpsychologie. In A. R. Buss (Ed.), Psychology in social context. New York: Irvington

Day, A., \& White, J. (2008). Ethical practice from the perspective of the forensic psychologist: Commentary on the uses and value of the Australian psychological society (2007) code of ethics. Australian Psychologist, 43(3), 186-193.

Filbert, K. M., \& Flynn, R. J. (2010). Developmental and cultural assets and resilient outcomes in first nations young people in care: An initial test of an explanatory model. Children and Youth Services Review, 32, 560-564.

Flanagan, J. (1954). The critical incident technique. Psychological Bulletin, 51, 327-358.

Hallett, D., Want, S. C., Chandler, M. J., Koopman, L. L., Flores, J., \& Gehrke, E. C. (2008). Identity in flux: Ethnic self-identification, and school attrition in Canadian aboriginal youth. Journal of Applied Developmental Psychology, 29, 62-75.

Kim, U., \& Berry, J. (Eds.). (1993). Indigenous psychologies: Research and experience in cultural context. London: Sage Publishing. 
Kingsley, J. Y., Phillips, R., Townsend, M., \& Henderson-Wilson, C. (2010). Using a qualitative approach to use research to build trust between a non-aboriginal researcher and aboriginal participants (Australia). Qualitative Research Journal, 10(1), 2-12.

McCormick, R. (1996). Culturally appropriate means and ends of counseling as described by the first nations people of British Columbia. International Journal for the Advancement of Counseling, 18, 163-172.

McKeough, A., Bird, S., Tourigny, E., Romaine, A., Graham, S., Ottmann, J., \& Jeary, J. (2008). Storytelling as a foundation to literacy development for aboriginal Children: Culturally and developmentally appropriate practices. Canadian Psychology, 49(2), 148-154.

NHMRC (National Health and Medical Research Council). (2003). Values and ethics: Guidelines for ethical conduct in aboriginal and Torres Strait islander health research. Canberra: National Health and Medical Research Council.

Norman, I. J., Redfern, S. J., Tomalin, D. A., \& Oliver, S. (1992). Developing Flanagan's critical incident technique to elicit indicators of high and low quality nursing care from patients and their nurses. Journal of Advanced Nursing, 17(5), 590-600.

Pedersen, A., \& Barlow, F. K. (2008). Theory to social action: A university-based strategy targeting prejudice against aboriginal australians. Australian Psychologist, 43(3), 148-159.

Rice, P. L., \& Ezzy, D. (1999). Qualitative research methods: A health focus. Oxford: Oxford University Press.

Schluter, J., Seaton, P., \& Chaboyer, W. (2008). Critical incident technique: A user's guide for nurse researchers. Journal of Advanced Nursing, 61(1), 107-114.

Woolsey, L. K. (1986). The critical incident technique: An innovative qualitative method of research. Canadian Journal of Counseling, 20(4), 242-254.

Wundt, W. (1916). Elements of folk psychology: Outlines of a psychological history of development of mankind (E. L. Schaub Trans.). London: George Allen and Unwin. 